Acta Crystallographica Section E

Structure Reports

Online

ISSN 1600-5368

\section{6-Methyl-2-pyridone pentahydrate}

\section{William Clegg* and Gary S. Nichol}

School of Natural Sciences (Chemistry), Bedson Building, University of Newcastle, Newcastle upon Tyne NE1 7RU, England

Correspondence e-mail: w.clegg@ncl.ac.uk

\section{Key indicators}

Single-crystal X-ray study

$T=150 \mathrm{~K}$

Mean $\sigma(\mathrm{C}-\mathrm{C})=0.003 \AA$

$R$ factor $=0.047$

$w R$ factor $=0.133$

Data-to-parameter ratio $=12.8$

For details of how these key indicators were automatically derived from the article, see http://journals.iucr.org/e.

Crystals of the title compound, $\mathrm{C}_{6} \mathrm{H}_{7} \mathrm{NO} \cdot 5 \mathrm{H}_{2} \mathrm{O}$, were grown over a period of several weeks from an aqueous solution of the commercial compound. The molecule crystallizes in space group $P \overline{1}$ and there are two independent 6-methyl-2-pyridone (Hmhp) molecules in the asymmetric unit, together with ten molecules of water. Packing diagrams reveal stacks of hydrogen-bonded Hmhp dimers surrounded by channels of water molecules. The Hmhp molecules pack with face-to-face $\pi-\pi$ stacking, a common feature of pyridone crystal structures. Each water molecule serves twice as hydrogen-bond donor and twice as acceptor, and is thus pseudo-tetrahedral. The water molecules are arranged in hydrogen-bonded five- and six-membered rings and the rings are fused together, with the five-membered rings adopting an envelope conformation and the six-membered rings adopting either a chair or boat conformation. This structure is further evidence that Hmhp exists in the solid state as the pyridone tautomer and not the pyridinol tautomer.

\title{
Comment
}

The molecule 2-hydroxypyridine and the family of 6-substituted derivatives have been extensively used as ligands in transition metal coordination chemistry, and a detailed review has been published (Rawson \& Winpenny, 1995). There is also much interest in the chemistry of the ligands themselves, in particular, the keto-enol tautomerism which is observed in the gas phase and in solution.<smiles>Cc1cccc(O)n1</smiles>

pyridinol
Received 22 July 2004 Accepted 27 July 2004 Online 31 July 2004<smiles>Cc1cccc(=O)[nH]1</smiles>

(I)
This tautomerism has been known since 1907 (Baker \& Baly, 1907) and has been comprehensively investigated in solution by IR spectroscopy (Gibson et al., 1955; Katritzky et al., 1967; Mason, 1957) and nuclear magnetic resonance spectroscopy (Coburn \& Dudek, 1968), and in the gas phase by IR spectroscopy (King et al., 1972; Beak \& Fry, 1973) and UV-vis spectroscopy (Beak et al., 1976). Various theoretical studies have also been reported (Beak \& Covington, 1978; Beak et al., 1980; Parchment et al., 1991; Wong et al., 1992). 


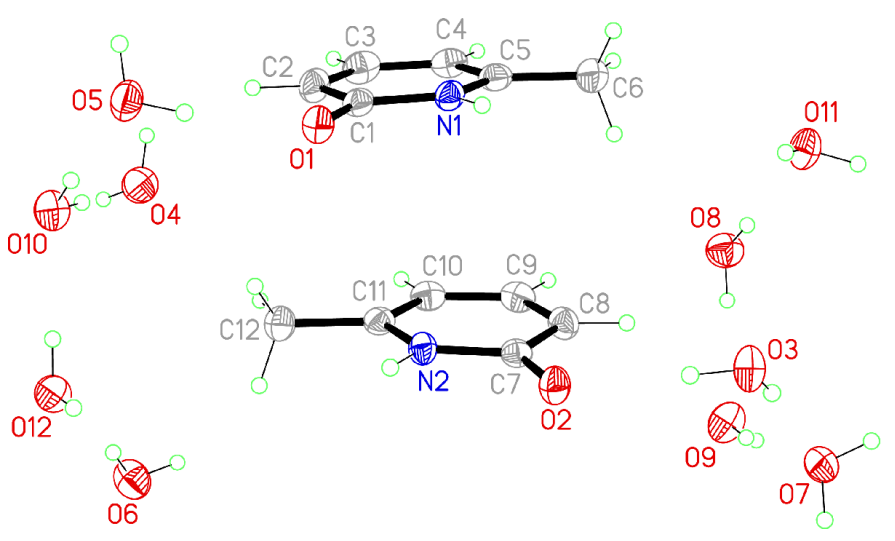

Figure 1

The asymmetric unit of (I), with displacement ellipsoids drawn at the $50 \%$ probability level. $\mathrm{H}$ atoms are represented as small spheres of arbitrary size.

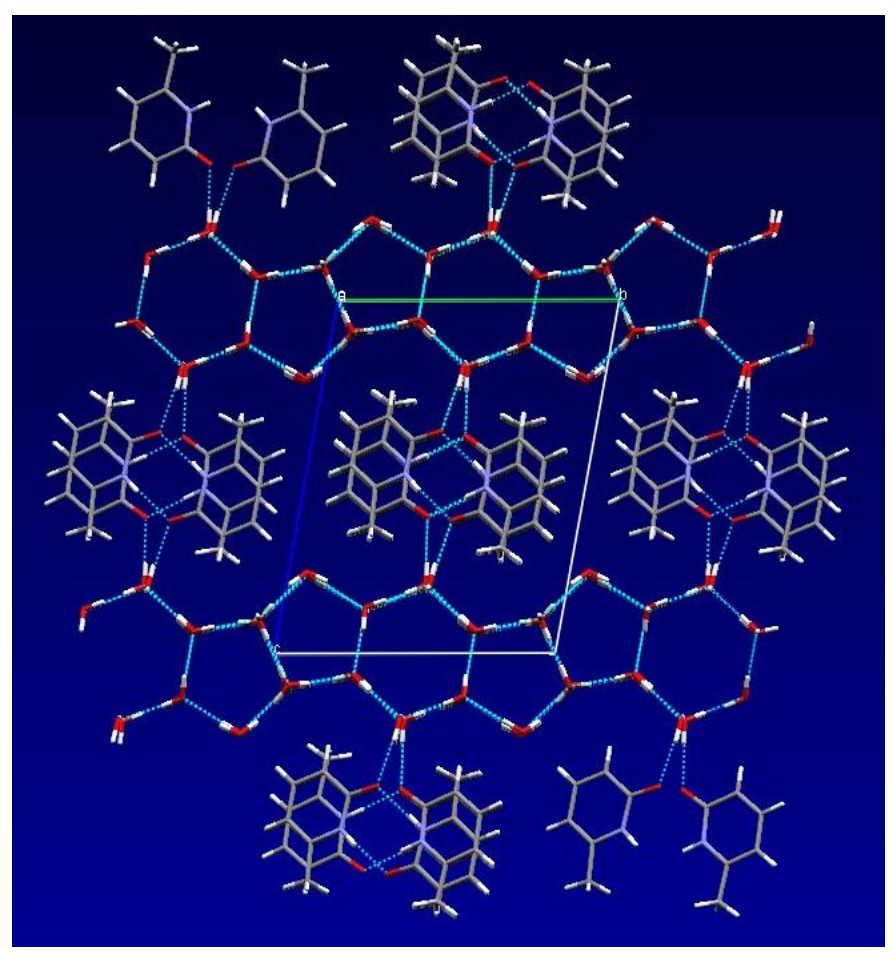

Figure 2

Projection along the $a$ axis, with hydrogen bonds in light blue, showing also the $\pi-\pi$ stacking.

Principal factors influencing the position of this equilibrium include solvent effects (polarity and $\mathrm{pH}$ ) and substituent effects (position on the ring and electron-donating/withdrawing influence). Substituents at position 6 have the greatest effect; electron-withdrawing substituents are seen to drive the equilibrium towards the pyridinol tautomer, whereas electrondonating substituents favour the pyridone tautomer. The rationale behind this has already been explained in terms of resonance stabilization and destabilization by the substituent (King et al., 1972).

Naturally, X-ray crystallography has played a key role in determining the preferred tautomers in the solid state. The structures of 6-chloro-2-hydroxypyridine (Kvick \& Olovsson, 1969) and 6-bromo-2-hydroxypyridine (Kvick, 1976) are

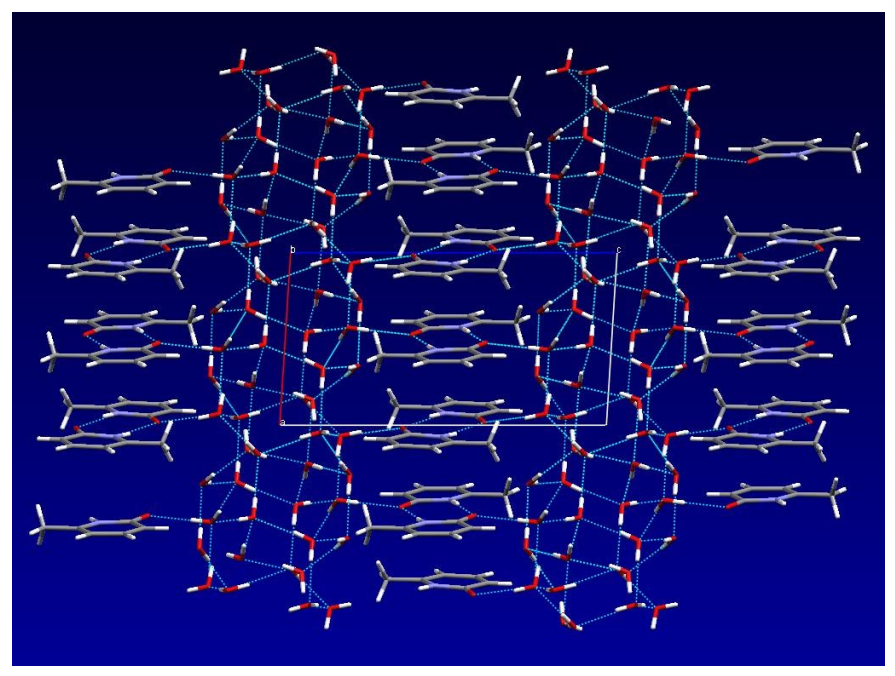

Figure 3

Projection along the $b$ axis, showing the hydrogen-bonding network involving the water molecules and the 6-methyl-2-pyridone dimer.

already known. Both have electron-withdrawing substituents and both crystallize as the pyridinol form, confirming the conclusions derived from spectroscopic evidence which predicted that they would be observed as the pyridinol tautomer. If there is no substituent, the molecule crystallizes as the pyridone form (Penfold, 1953).

However, crystallographic proof that electron-donating substituents generate a preference for the pyridone form has so far only been obtained via crystal structures of coordination compounds (Rawson \& Winpenny, 1995, and references therein) or co-crystallized with $(S)$-malic acid (Aakeröy et al., 2000). Unfortunately this is far from conclusive; a search of the Cambridge Structural Database (Version 5.25 plus two updates; Allen, 2002) for 6-methyl-2-pyridone, allowing all bonds to be of any type, returned 105 hits. Of these no fewer than 80 reported the ligand as the pyridinol form, rather than the pyridone form, and a handful of hits even contained mixtures of the two. In most cases, this is probably because the $\mathrm{C}-\mathrm{O}$ bond was too long to be considered as a genuine double bond. In addition, the ligand has been deprotonated in its complexes; there is no longer the possibility of determining the presence of an $\mathrm{O}-\mathrm{H}$ or $\mathrm{N}-\mathrm{H}$ bond and hence decide upon pyridone or pyridinol structure; these are now resonance forms rather than discrete tautomers.

We have determined the crystal structure of 6-methyl-2pyridone (Hmhp) as its pentahydrate, (I), crystallized from water (Fig. 1). There are two independent Hmhp molecules in the asymmetric unit and also ten molecules of water. Face-toface $\pi-\pi$ stacking, a common feature of pyridone crystal structures, is observed here. One Hmhp molecule lies above the other, with the methyl groups oriented in opposite directions, as shown in Fig. 2. Both molecules are essentially planar, except for the methyl $\mathrm{H}$ atoms. Their mean planes are approximately parallel and $3.30 \AA$ apart, just less than the sum of the van der Waals radii for two C atoms, which is $3.4 \AA$.

Packing diagrams of the structure show that Hmhp packs as hydrogen-bonded dimers; these then form stacks, separated by 
channels of hydrogen-bonded water molecules (Fig. 2). Each water molecule serves twice as hydrogen-bond donor and twice as acceptor, making four hydrogen bonds in total. This pseudo-tetrahedral arrangement means that the $\mathrm{O}$ atoms form five- and six-membered rings. These rings are fused together; the five-membered rings adopt an envelope conformation and the six-membered rings adopt either a chair or boat conformation. The hydrogen-bonding network involving the water molecules, and also the hydrogen bonding between the Hmhp molecules, is shown in Fig. 3.

The $\mathrm{C}-\mathrm{O}$ bond lengths are 1.272 (3) and 1.268 (3) $\AA$ for the two molecules and are in good agreement with those reported by Aakeröy et al. (2000) of 1.275 and $1.284 \AA$. These are a little on the long side for a $\mathrm{C}=\mathrm{O}$ double bond. However, the data clearly show the presence of an $\mathrm{H}$ atom bonded to each $\mathrm{N}$ atom, and these have been refined freely. From this result, together with the lack of significant residual electron density next to the $\mathrm{O}$ atoms, the molecule is unambiguously in the pyridone form.

\section{Experimental}

Commercially available 2-hydroxy-6-methylpyridine was obtained as a white powder. A sample was dissolved in distilled water with gentle heating and the sample vial stoppered. Storage in a cool cupboard resulted in large plate crystals growing over a period of several weeks.

\section{Crystal data}

$\mathrm{C}_{6} \mathrm{H}_{7} \mathrm{NO} \cdot 5 \mathrm{H}_{2} \mathrm{O}$
$M_{r}=199.21$
Triclinic, $P \overline{1}$
$a=7.5134(19) \AA$
$b=11.261(3) \AA$
$c=13.859(4) \AA$
$\alpha=99.674(4)^{\circ}$
$\beta=90.793(5)^{\circ}$
$\gamma=105.622(4)^{\circ}$
$V=1111.0(5) \AA^{3}$

$Z=4$

$D_{x}=1.191 \mathrm{Mg} \mathrm{m}^{-3}$

Mo $K \alpha$ radiation

Cell parameters from 2910 reflections

$\theta=2.2-25.0^{\circ}$

$\mu=0.11 \mathrm{~mm}^{-1}$

$T=150(2) \mathrm{K}$

Plate, colourless

$0.20 \times 0.10 \times 0.01 \mathrm{~mm}$

\section{Data collection}

\section{Bruker SMART 1K CCD}

diffractometer

Thin-slice $\omega$ scans

Absorption correction: none

8151 measured reflections

3872 independent reflections

$$
\begin{aligned}
& 2768 \text { reflections with } I>2 \sigma(I) \\
& R_{\text {int }}=0.030 \\
& \theta_{\max }=25.0^{\circ} \\
& h=-8 \rightarrow 8 \\
& k=-13 \rightarrow 13 \\
& l=-16 \rightarrow 16
\end{aligned}
$$

\section{Refinement}

Refinement on $F^{2}$

$R\left[F^{2}>2 \sigma\left(F^{2}\right)\right]=0.048$

$w R\left(F^{2}\right)=0.133$

$S=1.04$

3872 reflections

303 parameters

$\mathrm{H}$ atoms treated by a mixture of independent and constrained refinement

\begin{tabular}{|c|c|c|c|c|}
\hline$D-\mathrm{H} \cdots A$ & $D-\mathrm{H}$ & $\mathrm{H} \cdots A$ & $D \cdots A$ & $D-\mathrm{H} \cdots A$ \\
\hline $\mathrm{O} 3-\mathrm{H} 1 \mathrm{O} \cdots \mathrm{O} 2$ & $0.816(9)$ & $1.876(10)$ & $2.690(2)$ & $175(3)$ \\
\hline $\mathrm{O} 3-\mathrm{H} 2 \mathrm{O} \cdots \mathrm{O} 7$ & $0.813(10)$ & $1.944(11)$ & $2.750(3)$ & $171(3)$ \\
\hline $\mathrm{O} 4-\mathrm{H} 4 \mathrm{O} \cdots \mathrm{O} 6^{\mathrm{i}}$ & $0.816(10)$ & $1.967(12)$ & $2.766(3)$ & $166(3)$ \\
\hline $\mathrm{O} 4-\mathrm{H} 3 \mathrm{O} \cdots \mathrm{O} 6^{\mathrm{ii}}$ & $0.815(10)$ & $1.985(12)$ & $2.789(3)$ & $169(2)$ \\
\hline $\mathrm{O} 5-\mathrm{H} 5 \mathrm{O} \cdots \mathrm{O} 1$ & $0.824(9)$ & $1.887(11)$ & $2.701(2)$ & $170(3)$ \\
\hline $\mathrm{O} 5-\mathrm{H} 6 \mathrm{O} \cdots \mathrm{O} 3^{\mathrm{iii}}$ & $0.822(10)$ & $1.939(11)$ & $2.751(3)$ & $169(2)$ \\
\hline $\mathrm{O} 6-\mathrm{H} 7 \mathrm{O} \cdots \mathrm{O} 12$ & $0.813(10)$ & $1.935(10)$ & $2.744(3)$ & $173(3)$ \\
\hline $\mathrm{O} 6-\mathrm{H} 8 \mathrm{O} \cdots \mathrm{O} 8^{\mathrm{iv}}$ & $0.813(10)$ & $1.953(10)$ & $2.760(3)$ & $172(3)$ \\
\hline $\mathrm{O} 7-\mathrm{H} 10 \mathrm{O} \cdots \mathrm{O} 12^{\mathrm{v}}$ & $0.814(10)$ & $1.943(11)$ & $2.754(2)$ & $174(3)$ \\
\hline $\mathrm{O} 7-\mathrm{H} 9 \mathrm{O} \cdots \mathrm{O} 11^{\mathrm{vi}}$ & $0.810(10)$ & $1.965(10)$ & $2.774(3)$ & $176(3)$ \\
\hline $\mathrm{O} 8-\mathrm{H} 11 \mathrm{O} \cdots \mathrm{O} 11$ & $0.820(10)$ & $2.006(10)$ & $2.823(3)$ & $175(3)$ \\
\hline $\mathrm{O} 8-\mathrm{H} 12 \mathrm{O} \cdots \mathrm{O} 9$ & $0.818(10)$ & $1.963(10)$ & $2.780(3)$ & $177(3)$ \\
\hline $\mathrm{O} 9-\mathrm{H} 13 \mathrm{O} \cdots \mathrm{O} 7$ & $0.824(10)$ & $1.990(12)$ & $2.801(3)$ & $168(3)$ \\
\hline $\mathrm{O} 9-\mathrm{H} 14 \mathrm{O} \cdots \mathrm{O} 4^{\mathrm{iv}}$ & $0.814(10)$ & $2.019(12)$ & $2.816(3)$ & $166(3)$ \\
\hline $\mathrm{O} 10-\mathrm{H} 16 \mathrm{O} \cdots \mathrm{O} 4$ & $0.821(9)$ & $1.919(10)$ & $2.739(3)$ & $176(3)$ \\
\hline $\mathrm{O} 10-\mathrm{H} 15 \mathrm{O} \cdots \mathrm{O} 5$ & $0.822(10)$ & $1.917(11)$ & $2.736(3)$ & $174(2)$ \\
\hline $\mathrm{O} 11-\mathrm{H} 17 \mathrm{O} \cdots \mathrm{O} 10^{\mathrm{v}}$ & $0.817(10)$ & $1.940(10)$ & $2.752(2)$ & $173(3)$ \\
\hline $\mathrm{O} 11-\mathrm{H} 18 \mathrm{O} \cdots \mathrm{O} 5^{\mathrm{iii}}$ & $0.818(10)$ & $1.927(10)$ & $2.743(2)$ & $175(3)$ \\
\hline $\mathrm{O} 12-\mathrm{H} 19 \mathrm{O} \cdots \mathrm{O}^{\mathrm{vii}}$ & $0.820(10)$ & $1.907(10)$ & $2.726(2)$ & $177(3)$ \\
\hline $\mathrm{O} 12-\mathrm{H} 20 \mathrm{O} \cdots \mathrm{O} 10$ & $0.818(9)$ & $1.926(10)$ & $2.743(3)$ & $177(3)$ \\
\hline $\mathrm{N} 1-\mathrm{H} 1 \mathrm{~N} \cdots \mathrm{O} 1^{\mathrm{iii}}$ & $0.81(3)$ & $2.00(3)$ & $2.798(3)$ & $171(3)$ \\
\hline $\mathrm{N} 2-\mathrm{H} 2 \mathrm{~N} \cdots \mathrm{O} 2^{\mathrm{vii}}$ & $0.87(3)$ & $1.92(3)$ & $2.783(3)$ & $175(2)$ \\
\hline
\end{tabular}

\section{Table 1}

Selected bond distances $(\AA)$.

\begin{tabular}{llll}
\hline $\mathrm{O} 1-\mathrm{C} 1$ & $1.272(3)$ & $\mathrm{N} 1-\mathrm{H} 1 \mathrm{~N}$ & $0.81(3)$ \\
$\mathrm{O} 2-\mathrm{C} 7$ & $1.268(3)$ & $\mathrm{N} 2-\mathrm{H} 2 \mathrm{~N}$ & $0.87(3)$ \\
\hline
\end{tabular}

Table 2

Hydrogen-bonding geometry $\left(\AA{ }^{\circ}\right)$.

Methyl $\mathrm{H}$ atoms were positioned geometrically $(\mathrm{C}-\mathrm{H}=0.98 \AA$ ) and refined as riding, with free rotation about the $\mathrm{C}-\mathrm{C}$ bond, and with $U_{\text {iso }}(\mathrm{H})=1.5 U_{\text {eq }}(\mathrm{C})$. Aromatic $\mathrm{H}$ atoms were also positioned geometrically $(\mathrm{C}-\mathrm{H}=0.95 \AA)$ and refined as riding, with $U_{\text {iso }}(\mathrm{H})=$ $1.2 U_{\text {eq }}(\mathrm{C}) . \mathrm{H}$ atoms bonded to $\mathrm{N}$ and $\mathrm{O}$ atoms were found in a difference map and their positions were refined, with $U_{\text {iso }}(\mathrm{H})=$ $1.2 U_{\text {eq }}(\mathrm{N}, \mathrm{O})$. Water $\mathrm{O}-\mathrm{H}$ distances were restrained to 0.82 (1) $\AA$ and $\mathrm{H} \cdots \mathrm{H}$ distances restrained to 1.35 (2) $\AA$, but $\mathrm{N}-\mathrm{H}$ distances were not restrained.

Data collection: SMART (Bruker, 2001); cell refinement: SAINT (Bruker, 2001); data reduction: $S A I N T$; $\operatorname{program}(\mathrm{s})$ used to solve structure: SIR92 (Altomare et al., 1993); program(s) used to refine structure: SHELXTL (Sheldrick, 2001); molecular graphics: SHELXTL and MERCURY (Version 1.2; Bruno et al., 2002); software used to prepare material for publication: SHELXTL and local programs.

We thank the EPSRC for financial support.

\section{References}

Aakeröy, C. B., Beatty, A. M., Nieuwenhuyzen, M. \& Zou, M. (2000), Tetrahedron, 56, 6693-6699.

Allen, F. H. (2002). Acta Cryst. B58, 380-388.

Altomare, A., Cascarano, G., Giacovazzo, C. \& Guagliardi, A. (1993). J. Appl. Cryst. 26, 343-350.

Baker, F. \& Baly, E. C. C. (1907). J. Chem. Soc. pp. 1122-1132.

Beak, P. \& Fry, F. S. (1973). J. Am. Chem. Soc. 95, 1700-1702.

Beak, P., Fry, F. S., Lee, J. \& Steele, F. (1976). J. Am. Chem. Soc. 98, 171-179.

Beak, P. \& Covington, J. B. (1978). J. Am. Chem. Soc. 100, 3961-3963.

Beak, P., Covington, J. B. \& White, J. M. (1980). J. Org. Chem. 45, 1347-1353.

Bruker (2001). SMART and SAINT. Bruker AXS Inc., Madison, Wisconsin, USA.

Bruno, I. J., Cole, J. C., Edgington, P. R., Kessler, M., Macrae, C. F., McCabe, P., Pearson, J. \& Taylor, R. (2002). Acta Cryst. B58, 389-397.

Coburn, R. A. \& Dudek, G. O. (1968). J. Phys. Chem. 72, 1177-1181.

Gibson, J. A., Kynaston, W. \& Lindsey, A. S. (1955). J. Chem. Soc. pp. 43404344.

Katritzky, A. R., Rowe, J. D. \& Roy, S. K. (1967). J. Chem. Soc. B, pp. 758-761.

King, S. S. T., Dilling, W. L \& Tefertiller, N. B. (1972). Tetrahedron, 28, 58595863.

Kvick, Å. (1976). Acta Cryst. B32, 220-224. 


\section{organic papers}

Kvick, Å. \& Olovsson, I. (1969). Ark. Kemi, 30, 71-80.

Mason, S. F. (1957). J. Chem. Soc. pp. 4874-4880.

Parchment, O. G., Hillier, I. H. \& Green, D. S. V. (1991). J. Chem. Soc. Perkin Trans. 2, pp. 799-802.

Penfold, B. R. (1953). Acta Cryst. 6, 591-600.
Rawson, J. M. \& Winpenny, R. E. P. (1995). Coord. Chem. Rev. 139, 313-374.

Sheldrick, G. M. (2001). SHELXTL. Version 6. Bruker AXS Inc., Madison, Wisconsin, USA.

Wong, M. W., Wiberg, K. B. \& Frisch, M. J. (1992). J. Am. Chem. Soc. 114 1645-1652. 


\section{supporting information}

Acta Cryst. (2004). E60, o1433-o1436 [https://doi.org/10.1107/S1600536804018367]

\section{6-Methyl-2-pyridone pentahydrate}

\section{William Clegg and Gary S. Nichol}

6-methyl-2-pyridone pentahydrate

Crystal data

$\mathrm{C}_{6} \mathrm{H}_{7} \mathrm{NO} \cdot 5 \mathrm{H}_{2} \mathrm{O}$

$M_{r}=199.21$

Triclinic, $P \overline{1}$

Hall symbol: -P 1

$a=7.5134(19) \AA$

$b=11.261(3) \AA$

$c=13.859(4) \AA$

$\alpha=99.674(4)^{\circ}$

$\beta=90.793(5)^{\circ}$

$\gamma=105.622(4)^{\circ}$

$V=1111.0(5) \AA^{3}$

\section{Data collection}

Bruker SMART 1K CCD diffractometer

Radiation source: sealed tube Graphite monochromator thin-slice $\omega$ scans

8151 measured reflections

3872 independent reflections

\section{Refinement}

Refinement on $F^{2}$

Least-squares matrix: full

$R\left[F^{2}>2 \sigma\left(F^{2}\right)\right]=0.048$

$w R\left(F^{2}\right)=0.133$

$S=1.04$

3872 reflections

303 parameters

30 restraints

Primary atom site location: structure-invariant direct methods
$Z=4$

$F(000)=432$

$D_{\mathrm{x}}=1.191 \mathrm{Mg} \mathrm{m}^{-3}$

Mo $K \alpha$ radiation, $\lambda=0.71073 \AA$

Cell parameters from 2910 reflections

$\theta=2.2-25.0^{\circ}$

$\mu=0.11 \mathrm{~mm}^{-1}$

$T=150 \mathrm{~K}$

Plate, colourless

$0.20 \times 0.10 \times 0.01 \mathrm{~mm}$

2768 reflections with $I>2 \sigma(I)$

$R_{\text {int }}=0.030$

$\theta_{\max }=25.0^{\circ}, \theta_{\min }=1.5^{\circ}$

$h=-8 \rightarrow 8$

$k=-13 \rightarrow 13$

$l=-16 \rightarrow 16$

\section{Special details}

Geometry. All e.s.d.'s (except the e.s.d. in the dihedral angle between two 1.s. planes) are estimated using the full covariance matrix. The cell e.s.d.'s are taken into account individually in the estimation of e.s.d.'s in distances, angles and torsion angles; correlations between e.s.d.'s in cell parameters are only used when they are defined by crystal symmetry. An approximate (isotropic) treatment of cell e.s.d.'s is used for estimating e.s.d.'s involving 1.s. planes. 
Refinement. Refinement of $F^{2}$ against ALL reflections. The weighted $R$-factor $w R$ and goodness of fit $S$ are based on $F^{2}$, conventional $R$-factors $R$ are based on $F$, with $F$ set to zero for negative $F^{2}$. The threshold expression of $F^{2}>\sigma\left(F^{2}\right)$ is used only for calculating $R$-factors(gt) etc. and is not relevant to the choice of reflections for refinement. $R$-factors based on $F^{2}$ are statistically about twice as large as those based on $F$, and $R$ - factors based on ALL data will be even larger.

Fractional atomic coordinates and isotropic or equivalent isotropic displacement parameters $\left(\hat{A}^{2}\right)$

\begin{tabular}{|c|c|c|c|c|}
\hline & $x$ & $y$ & $z$ & $U_{\text {iso }} * / U_{\text {eq }}$ \\
\hline $\mathrm{O} 1$ & $1.0162(2)$ & $0.45299(15)$ & $0.37534(11)$ & $0.0261(4)$ \\
\hline $\mathrm{O} 2$ & $0.5241(2)$ & $0.45850(14)$ & $0.61329(11)$ & $0.0242(4)$ \\
\hline $\mathrm{O} 3$ & 0.5648 & $0.49525(16)$ & $0.81011(12)$ & $0.0301(4)$ \\
\hline $\mathrm{H} 1 \mathrm{O}$ & $0.551(4)$ & $0.488(2)$ & $0.7507(8)$ & $0.036^{*}$ \\
\hline $\mathrm{H} 2 \mathrm{O}$ & $0.504(3)$ & $0.4343(17)$ & 0.8307 (17) & $0.036^{*}$ \\
\hline $\mathrm{O} 4$ & 0.8545 & $0.07316(17)$ & $0.09483(13)$ & $0.0351(4)$ \\
\hline $\mathrm{H} 4 \mathrm{O}$ & $0.9648(16)$ & $0.080(3)$ & $0.1036(19)$ & $0.042 *$ \\
\hline $\mathrm{H} 3 \mathrm{O}$ & $0.818(3)$ & $0.032(2)$ & 0.0407 (11) & $0.042 *$ \\
\hline $\mathrm{O} 5$ & $1.0637(2)$ & $0.49021(17)$ & $0.18880(12)$ & $0.0303(4)$ \\
\hline $\mathrm{H} 5 \mathrm{O}$ & $1.041(3)$ & $0.485(3)$ & $0.2461(10)$ & $0.036^{*}$ \\
\hline $\mathrm{H} 6 \mathrm{O}$ & $1.1729(17)$ & $0.493(3)$ & $0.1813(18)$ & $0.036^{*}$ \\
\hline O6 & $0.2236(3)$ & $0.07603(17)$ & $0.09106(14)$ & $0.0360(4)$ \\
\hline $\mathrm{H} 7 \mathrm{O}$ & $0.300(3)$ & $0.1411(16)$ & $0.087(2)$ & $0.043 *$ \\
\hline $\mathrm{H} 8 \mathrm{O}$ & $0.248(4)$ & $0.040(2)$ & 0.1337 (16) & $0.043 *$ \\
\hline $\mathrm{O} 7$ & $0.3284(2)$ & $0.29902(17)$ & $0.87828(12)$ & $0.0306(4)$ \\
\hline $\mathrm{H} 10 \mathrm{O}$ & $0.376(3)$ & $0.302(3)$ & $0.9323(11)$ & $0.037 *$ \\
\hline H9O & $0.2214(17)$ & $0.301(3)$ & 0.8794 (18) & $0.037^{*}$ \\
\hline $\mathrm{O} 8$ & $0.7141(3)$ & $0.06853(17)$ & $0.77671(14)$ & $0.0352(4)$ \\
\hline $\mathrm{H} 11 \mathrm{O}$ & $0.779(3)$ & $0.1365(15)$ & 0.8051 (19) & $0.042 *$ \\
\hline $\mathrm{H} 12 \mathrm{O}$ & $0.6052(15)$ & $0.068(2)$ & $0.777(2)$ & $0.042 *$ \\
\hline O9 & $0.3437(3)$ & $0.06773(17)$ & 0.77195 (13) & $0.0365(5)$ \\
\hline $\mathrm{H} 13 \mathrm{O}$ & $0.332(4)$ & $0.1373(13)$ & 0.7956 (19) & $0.044 *$ \\
\hline $\mathrm{H} 14 \mathrm{O}$ & $0.290(4)$ & $0.0167(18)$ & $0.8045(18)$ & $0.044 *$ \\
\hline $\mathrm{O} 10$ & 0.8290 & $0.30035(16)$ & $0.05951(12)$ & $0.0310(4)$ \\
\hline $\mathrm{H} 16 \mathrm{O}$ & $0.837(4)$ & $0.2334(13)$ & $0.0726(18)$ & $0.037 *$ \\
\hline $\mathrm{H} 15 \mathrm{O}$ & $0.897(3)$ & $0.3607(15)$ & 0.0967 (16) & $0.037^{*}$ \\
\hline O11 & $0.9578(3)$ & $0.29564(16)$ & $0.87435(12)$ & $0.0309(4)$ \\
\hline $\mathrm{H} 17 \mathrm{O}$ & $0.928(4)$ & $0.302(2)$ & $0.9311(9)$ & $0.037^{*}$ \\
\hline $\mathrm{H} 18 \mathrm{O}$ & $0.956(4)$ & $0.3591(16)$ & $0.8534(17)$ & $0.037^{*}$ \\
\hline $\mathrm{O} 12$ & $0.4631(2)$ & $0.29778(16)$ & $0.06383(12)$ & $0.0296(4)$ \\
\hline H19O & $0.452(3)$ & $0.3583(17)$ & $0.1031(16)$ & $0.036^{*}$ \\
\hline $\mathrm{H} 20 \mathrm{O}$ & $0.5709(18)$ & $0.295(2)$ & $0.0616(19)$ & $0.036^{*}$ \\
\hline N1 & $1.0683(3)$ & $0.35222(18)$ & $0.49688(14)$ & $0.0211(4)$ \\
\hline $\mathrm{H} 1 \mathrm{~N}$ & $1.052(3)$ & $0.408(2)$ & $0.5381(19)$ & $0.025 *$ \\
\hline $\mathrm{N} 2$ & 0.5668 & $0.35218(18)$ & $0.46533(13)$ & $0.0202(4)$ \\
\hline $\mathrm{H} 2 \mathrm{~N}$ & $0.545(3)$ & $0.414(2)$ & $0.4413(18)$ & $0.024 *$ \\
\hline $\mathrm{C} 1$ & $1.0545(3)$ & $0.3586(2)$ & $0.40031(16)$ & $0.0225(5)$ \\
\hline $\mathrm{C} 2$ & $1.0839(3)$ & $0.2550(2)$ & $0.33380(17)$ & $0.0271(5)$ \\
\hline $\mathrm{H} 2$ & 1.0788 & 0.2544 & 0.2652 & $0.033 *$ \\
\hline $\mathrm{C} 3$ & $1.1193(3)$ & $0.1572(2)$ & $0.36886(19)$ & $0.0308(6)$ \\
\hline
\end{tabular}




$\begin{array}{lllll}\text { H3 } & 1.1375 & 0.0883 & 0.3242 & 0.037^{*} \\ \text { C4 } & 1.1293(3) & 0.1567(2) & 0.46989(18) & 0.0272(5) \\ \text { H4 } & 1.1549 & 0.0881 & 0.4934 & 0.033^{*} \\ \text { C5 } & 1.1022(3) & 0.2546(2) & 0.53403(18) & 0.0257(5) \\ \text { C6 } & 1.1067(3) & 0.2653(2) & 0.64234(18) & 0.0310(6) \\ \text { H6A } & 1.1363 & 0.1920 & 0.6605 & 0.046^{*} \\ \text { H6B } & 0.9854 & 0.2692 & 0.6654 & 0.046^{*} \\ \text { H6C } & 1.2013 & 0.3415 & 0.6726 & 0.046^{*} \\ \text { C7 } & 0.5610(3) & 0.3630(2) & 0.56469(16) & 0.0205(5) \\ \text { C8 } & 0.5975(3) & 0.2633(2) & 0.60486(17) & 0.0242(5) \\ \text { H8 } & 0.5990 & 0.2662 & 0.6738 & 0.029^{*} \\ \text { C9 } & 0.6304(3) & 0.1636(2) & 0.54479(18) & 0.0258(5) \\ \text { H9 } & 0.6535 & 0.0972 & 0.5726 & 0.031^{*} \\ \text { C10 } & 0.6309(3) & 0.1570(2) & 0.44281(17) & 0.0246(5) \\ \text { H10 } & 0.6533 & 0.0866 & 0.4018 & 0.030^{*} \\ \text { C11 } & 0.5988(3) & 0.2525(2) & 0.40363(16) & 0.0218(5) \\ \text { C12 } & 0.5946(3) & 0.2590(2) & 0.29689(16) & 0.0274(5) \\ \text { H12A } & 0.6266 & 0.1862 & 0.2598 & 0.041^{*} \\ \text { H12B } & 0.6843 & 0.3360 & 0.2862 & 0.041^{*} \\ \text { H12C } & 0.4702 & 0.2589 & 0.2747 & 0.041^{*}\end{array}$

Atomic displacement parameters $\left(\AA^{2}\right)$

\begin{tabular}{lllllll}
\hline & $U^{11}$ & $U^{22}$ & $U^{33}$ & $U^{12}$ & $U^{13}$ & $U^{23}$ \\
\hline O1 & $0.0303(9)$ & $0.0274(9)$ & $0.0214(9)$ & $0.0090(7)$ & $0.0031(7)$ & $0.0051(7)$ \\
O2 & $0.0310(9)$ & $0.0236(9)$ & $0.0190(8)$ & $0.0102(7)$ & $0.0020(7)$ & $0.0024(7)$ \\
O3 & $0.0419(11)$ & $0.0291(10)$ & $0.0194(9)$ & $0.0092(8)$ & $0.0027(8)$ & $0.0054(7)$ \\
O4 & $0.0347(10)$ & $0.0345(11)$ & $0.0331(10)$ & $0.0064(9)$ & $0.0016(8)$ & $0.0025(8)$ \\
O5 & $0.0345(10)$ & $0.0356(10)$ & $0.0223(9)$ & $0.0104(9)$ & $0.0066(8)$ & $0.0077(8)$ \\
O6 & $0.0380(11)$ & $0.0288(10)$ & $0.0396(11)$ & $0.0037(8)$ & $-0.0039(9)$ & $0.0111(8)$ \\
O7 & $0.0310(10)$ & $0.0376(10)$ & $0.0259(9)$ & $0.0131(9)$ & $-0.0012(8)$ & $0.0077(8)$ \\
O8 & $0.0321(10)$ & $0.0330(10)$ & $0.0390(11)$ & $0.0088(9)$ & $0.0013(9)$ & $0.0027(8)$ \\
O9 & $0.0435(12)$ & $0.0292(10)$ & $0.0373(11)$ & $0.0103(9)$ & $0.0098(9)$ & $0.0058(8)$ \\
O10 & $0.0375(11)$ & $0.0267(10)$ & $0.0278(10)$ & $0.0086(8)$ & $0.0007(8)$ & $0.0027(8)$ \\
O11 & $0.0406(11)$ & $0.0309(10)$ & $0.0249(9)$ & $0.0129(8)$ & $0.0075(8)$ & $0.0098(8)$ \\
O12 & $0.0319(10)$ & $0.0301(10)$ & $0.0270(10)$ & $0.0114(8)$ & $0.0015(8)$ & $0.0005(7)$ \\
N1 & $0.0208(10)$ & $0.0217(10)$ & $0.0191(10)$ & $0.0052(8)$ & $0.0032(8)$ & $0.0003(8)$ \\
N2 & $0.0230(10)$ & $0.0193(10)$ & $0.0185(10)$ & $0.0048(8)$ & $0.0014(8)$ & $0.0057(8)$ \\
C1 & $0.0181(12)$ & $0.0241(13)$ & $0.0221(12)$ & $0.0011(10)$ & $0.0022(9)$ & $0.0029(10)$ \\
C2 & $0.0290(13)$ & $0.0282(13)$ & $0.0207(12)$ & $0.0057(11)$ & $0.0039(10)$ & $-0.0016(10)$ \\
C3 & $0.0287(14)$ & $0.0255(13)$ & $0.0353(15)$ & $0.0076(11)$ & $0.0062(11)$ & $-0.0030(11)$ \\
C4 & $0.0225(13)$ & $0.0236(13)$ & $0.0364(15)$ & $0.0065(10)$ & $0.0038(10)$ & $0.0076(11)$ \\
C5 & $0.0182(12)$ & $0.0286(13)$ & $0.0305(13)$ & $0.0042(10)$ & $0.0023(10)$ & $0.0093(10)$ \\
C6 & $0.0289(14)$ & $0.0387(15)$ & $0.0274(14)$ & $0.0101(12)$ & $0.0029(11)$ & $0.0107(11)$ \\
C7 & $0.0160(11)$ & $0.0206(12)$ & $0.0220(12)$ & $0.0000(9)$ & $0.0000(9)$ & $0.0043(9)$ \\
C8 & $0.0243(13)$ & $0.0249(13)$ & $0.0234(12)$ & $0.0047(10)$ & $-0.0003(10)$ & $0.0079(10)$ \\
C9 & $0.0233(12)$ & $0.0213(12)$ & $0.0329(14)$ & $0.0040(10)$ & $-0.0004(10)$ & $0.0087(10)$ \\
C10 & $0.0221(12)$ & $0.0194(12)$ & $0.0299(13)$ & $0.0038(10)$ & $0.0009(10)$ & $0.0010(10)$
\end{tabular}




$\begin{array}{lllllll}\mathrm{C} 11 & 0.0173(11) & 0.0222(12) & 0.0218(12) & 0.0015(9) & 0.0003(9) & -0.0006(9) \\ \mathrm{C} 12 & 0.0290(13) & 0.0311(14) & 0.0212(12) & 0.0086(11) & 0.0024(10) & 0.0016(10)\end{array}$

Geometric parameters $\left(A,{ }^{\circ}\right)$

\begin{tabular}{|c|c|c|c|}
\hline $\mathrm{O} 1-\mathrm{C} 1$ & $1.272(3)$ & $\mathrm{N} 2-\mathrm{C} 7$ & $1.364(3)$ \\
\hline $\mathrm{O} 2-\mathrm{C} 7$ & $1.268(3)$ & $\mathrm{N} 2-\mathrm{C} 11$ & $1.370(3)$ \\
\hline $\mathrm{O} 3-\mathrm{H} 1 \mathrm{O}$ & $0.816(9)$ & $\mathrm{N} 2-\mathrm{H} 2 \mathrm{~N}$ & $0.87(3)$ \\
\hline $\mathrm{O} 3-\mathrm{H} 2 \mathrm{O}$ & $0.813(10)$ & $\mathrm{C} 1-\mathrm{C} 2$ & $1.429(3)$ \\
\hline $\mathrm{O} 4-\mathrm{H} 4 \mathrm{O}$ & $0.816(10)$ & $\mathrm{C} 2-\mathrm{C} 3$ & $1.362(3)$ \\
\hline $\mathrm{O} 4-\mathrm{H} 3 \mathrm{O}$ & $0.815(10)$ & $\mathrm{C} 2-\mathrm{H} 2$ & 0.950 \\
\hline $\mathrm{O} 5-\mathrm{H} 5 \mathrm{O}$ & $0.824(9)$ & $\mathrm{C} 3-\mathrm{C} 4$ & $1.402(4)$ \\
\hline $\mathrm{O} 5-\mathrm{H} 6 \mathrm{O}$ & $0.822(10)$ & $\mathrm{C} 3-\mathrm{H} 3$ & 0.950 \\
\hline $\mathrm{O} 6-\mathrm{H} 7 \mathrm{O}$ & $0.813(10)$ & $\mathrm{C} 4-\mathrm{C} 5$ & $1.358(3)$ \\
\hline $\mathrm{O} 6-\mathrm{H} 8 \mathrm{O}$ & $0.813(10)$ & $\mathrm{C} 4-\mathrm{H} 4$ & 0.950 \\
\hline $\mathrm{O} 7-\mathrm{H} 10 \mathrm{O}$ & $0.814(10)$ & $\mathrm{C} 5-\mathrm{C} 6$ & $1.485(3)$ \\
\hline $\mathrm{O} 7-\mathrm{H} 9 \mathrm{O}$ & $0.810(10)$ & C6-H6A & 0.980 \\
\hline $\mathrm{O} 8-\mathrm{H} 11 \mathrm{O}$ & $0.820(10)$ & C6-H6B & 0.980 \\
\hline $\mathrm{O} 8-\mathrm{H} 12 \mathrm{O}$ & $0.818(10)$ & $\mathrm{C} 6-\mathrm{H} 6 \mathrm{C}$ & 0.980 \\
\hline $\mathrm{O} 9-\mathrm{H} 13 \mathrm{O}$ & $0.824(10)$ & $\mathrm{C} 7-\mathrm{C} 8$ & $1.420(3)$ \\
\hline $\mathrm{O} 9-\mathrm{H} 14 \mathrm{O}$ & $0.814(10)$ & $\mathrm{C} 8-\mathrm{C} 9$ & $1.362(3)$ \\
\hline $\mathrm{O} 10-\mathrm{H} 16 \mathrm{O}$ & $0.821(9)$ & $\mathrm{C} 8-\mathrm{H} 8$ & 0.950 \\
\hline $\mathrm{O} 10-\mathrm{H} 15 \mathrm{O}$ & $0.822(10)$ & $\mathrm{C} 9-\mathrm{C} 10$ & $1.403(3)$ \\
\hline $\mathrm{O} 11-\mathrm{H} 17 \mathrm{O}$ & $0.817(10)$ & $\mathrm{C} 9-\mathrm{H} 9$ & 0.950 \\
\hline $\mathrm{O} 11-\mathrm{H} 18 \mathrm{O}$ & $0.818(10)$ & $\mathrm{C} 10-\mathrm{C} 11$ & $1.357(3)$ \\
\hline $\mathrm{O} 12-\mathrm{H} 19 \mathrm{O}$ & $0.820(10)$ & $\mathrm{C} 10-\mathrm{H} 10$ & 0.950 \\
\hline $\mathrm{O} 12-\mathrm{H} 20 \mathrm{O}$ & $0.818(9)$ & $\mathrm{C} 11-\mathrm{C} 12$ & $1.494(3)$ \\
\hline $\mathrm{N} 1-\mathrm{C} 1$ & $1.356(3)$ & $\mathrm{C} 12-\mathrm{H} 12 \mathrm{~A}$ & 0.980 \\
\hline $\mathrm{N} 1-\mathrm{C} 5$ & $1.369(3)$ & $\mathrm{C} 12-\mathrm{H} 12 \mathrm{~B}$ & 0.980 \\
\hline $\mathrm{N} 1-\mathrm{H} 1 \mathrm{~N}$ & $0.81(3)$ & $\mathrm{C} 12-\mathrm{H} 12 \mathrm{C}$ & 0.980 \\
\hline $\mathrm{H} 1 \mathrm{O}-\mathrm{O} 3-\mathrm{H} 2 \mathrm{O}$ & $113(2)$ & $\mathrm{C} 4-\mathrm{C} 5-\mathrm{C} 6$ & $125.2(2)$ \\
\hline $\mathrm{H} 4 \mathrm{O}-\mathrm{O} 4-\mathrm{H} 3 \mathrm{O}$ & $109(2)$ & $\mathrm{N} 1-\mathrm{C} 5-\mathrm{C} 6$ & $116.7(2)$ \\
\hline $\mathrm{H} 5 \mathrm{O}-\mathrm{O} 5-\mathrm{H} 6 \mathrm{O}$ & $109(2)$ & $\mathrm{C} 5-\mathrm{C} 6-\mathrm{H} 6 \mathrm{~A}$ & 109.5 \\
\hline $\mathrm{H} 7 \mathrm{O}-\mathrm{O} 6-\mathrm{H} 8 \mathrm{O}$ & $115(2)$ & $\mathrm{C} 5-\mathrm{C} 6-\mathrm{H} 6 \mathrm{~B}$ & 109.5 \\
\hline $\mathrm{H} 10 \mathrm{O}-\mathrm{O} 7-\mathrm{H} 9 \mathrm{O}$ & $113(2)$ & $\mathrm{H} 6 \mathrm{~A}-\mathrm{C} 6-\mathrm{H} 6 \mathrm{~B}$ & 109.5 \\
\hline $\mathrm{H} 11 \mathrm{O}-\mathrm{O} 8-\mathrm{H} 12 \mathrm{O}$ & $110(2)$ & $\mathrm{C} 5-\mathrm{C} 6-\mathrm{H} 6 \mathrm{C}$ & 109.5 \\
\hline $\mathrm{H} 13 \mathrm{O}-\mathrm{O} 9-\mathrm{H} 14 \mathrm{O}$ & $109(2)$ & $\mathrm{H} 6 \mathrm{~A}-\mathrm{C} 6-\mathrm{H} 6 \mathrm{C}$ & 109.5 \\
\hline $\mathrm{H} 16 \mathrm{O}-\mathrm{O} 10-\mathrm{H} 15 \mathrm{O}$ & $112(2)$ & $\mathrm{H} 6 \mathrm{~B}-\mathrm{C} 6-\mathrm{H} 6 \mathrm{C}$ & 109.5 \\
\hline $\mathrm{H} 17 \mathrm{O}-\mathrm{O} 11-\mathrm{H} 18 \mathrm{O}$ & $110(2)$ & $\mathrm{O} 2-\mathrm{C} 7-\mathrm{N} 2$ & $118.9(2)$ \\
\hline $\mathrm{H} 19 \mathrm{O}-\mathrm{O} 12-\mathrm{H} 20 \mathrm{O}$ & $112(2)$ & $\mathrm{O} 2-\mathrm{C} 7-\mathrm{C} 8$ & $125.5(2)$ \\
\hline $\mathrm{C} 1-\mathrm{N} 1-\mathrm{C} 5$ & $125.5(2)$ & $\mathrm{N} 2-\mathrm{C} 7-\mathrm{C} 8$ & $115.7(2)$ \\
\hline $\mathrm{C} 1-\mathrm{N} 1-\mathrm{H} 1 \mathrm{~N}$ & $120.5(18)$ & $\mathrm{C} 9-\mathrm{C} 8-\mathrm{C} 7$ & $120.1(2)$ \\
\hline $\mathrm{C} 5-\mathrm{N} 1-\mathrm{H} 1 \mathrm{~N}$ & $114.0(18)$ & $\mathrm{C} 9-\mathrm{C} 8-\mathrm{H} 8$ & 119.9 \\
\hline $\mathrm{C} 7-\mathrm{N} 2-\mathrm{C} 11$ & $125.1(2)$ & $\mathrm{C} 7-\mathrm{C} 8-\mathrm{H} 8$ & 119.9 \\
\hline $\mathrm{C} 7-\mathrm{N} 2-\mathrm{H} 2 \mathrm{~N}$ & $115.2(16)$ & $\mathrm{C} 8-\mathrm{C} 9-\mathrm{C} 10$ & $121.4(2)$ \\
\hline $\mathrm{C} 11-\mathrm{N} 2-\mathrm{H} 2 \mathrm{~N}$ & $119.8(16)$ & $\mathrm{C} 8-\mathrm{C} 9-\mathrm{H} 9$ & 119.3 \\
\hline $\mathrm{O} 1-\mathrm{C} 1-\mathrm{N} 1$ & $119.2(2)$ & $\mathrm{C} 10-\mathrm{C} 9-\mathrm{H} 9$ & 119.3 \\
\hline
\end{tabular}




$\begin{array}{ll}\mathrm{O} 1-\mathrm{C} 1-\mathrm{C} 2 & 125.0(2) \\ \mathrm{N} 1-\mathrm{C} 1-\mathrm{C} 2 & 115.7(2) \\ \mathrm{C} 3-\mathrm{C} 2-\mathrm{C} 1 & 119.9(2) \\ \mathrm{C} 3-\mathrm{C} 2-\mathrm{H} 2 & 120.0 \\ \mathrm{C} 1-\mathrm{C} 2-\mathrm{H} 2 & 120.0 \\ \mathrm{C} 2-\mathrm{C} 3-\mathrm{C} 4 & 121.0(2) \\ \mathrm{C} 2-\mathrm{C} 3-\mathrm{H} 3 & 119.5 \\ \mathrm{C} 4-\mathrm{C} 3-\mathrm{H} 3 & 119.5 \\ \mathrm{C} 5-\mathrm{C} 4-\mathrm{C} 3 & 119.7(2) \\ \mathrm{C} 5-\mathrm{C} 4-\mathrm{H} 4 & 120.1 \\ \mathrm{C} 3-\mathrm{C} 4-\mathrm{H} 4 & 120.1 \\ \mathrm{C} 4-\mathrm{C} 5-\mathrm{N} 1 & 118.1(2) \\ & \\ \mathrm{C} 5-\mathrm{N} 1-\mathrm{C} 1-\mathrm{O} 1 & 177.6(2) \\ \mathrm{C} 5-\mathrm{N} 1-\mathrm{C} 1-\mathrm{C} 2 & -1.9(3) \\ \mathrm{O} 1-\mathrm{C} 1-\mathrm{C} 2-\mathrm{C} 3 & -178.2(2) \\ \mathrm{N} 1-\mathrm{C} 1-\mathrm{C} 2-\mathrm{C} 3 & 1.3(3) \\ \mathrm{C} 1-\mathrm{C} 2-\mathrm{C} 3-\mathrm{C} 4 & -0.6(4) \\ \mathrm{C} 2-\mathrm{C} 3-\mathrm{C} 4-\mathrm{C} 5 & 0.4(4) \\ \mathrm{C} 3-\mathrm{C} 4-\mathrm{C} 5-\mathrm{N} 1 & -0.9(3) \\ \mathrm{C} 3-\mathrm{C} 4-\mathrm{C} 5-\mathrm{C} 6 & 179.2(2) \\ \mathrm{C} 1-\mathrm{N} 1-\mathrm{C} 5-\mathrm{C} 4 & 1.8(3) \\ \mathrm{C} 1-\mathrm{N} 1-\mathrm{C} 5-\mathrm{C} 6 & -178.3(2) \\ \end{array}$

$\begin{array}{ll}\mathrm{C} 11-\mathrm{C} 10-\mathrm{C} 9 & 119.0(2) \\ \mathrm{C} 11-\mathrm{C} 10-\mathrm{H} 10 & 120.5 \\ \mathrm{C} 9-\mathrm{C} 10-\mathrm{H} 10 & 120.5 \\ \mathrm{C} 10-\mathrm{C} 11-\mathrm{N} 2 & 118.7(2) \\ \mathrm{C} 10-\mathrm{C} 11-\mathrm{C} 12 & 125.3(2) \\ \mathrm{N} 2-\mathrm{C} 11-\mathrm{C} 12 & 116.0(2) \\ \mathrm{C} 11-\mathrm{C} 12-\mathrm{H} 12 \mathrm{~A} & 109.5 \\ \mathrm{C} 11-\mathrm{C} 12-\mathrm{H} 12 \mathrm{~B} & 109.5 \\ \mathrm{H} 12 \mathrm{~A}-\mathrm{C} 12-\mathrm{H} 12 \mathrm{~B} & 109.5 \\ \mathrm{C} 11-\mathrm{C} 12-\mathrm{H} 12 \mathrm{C} & 109.5 \\ \mathrm{H} 12 \mathrm{~A}-\mathrm{C} 12-\mathrm{H} 12 \mathrm{C} & 109.5 \\ \mathrm{H} 12 \mathrm{~B}-\mathrm{C} 12-\mathrm{H} 12 \mathrm{C} & 109.5 \\ & \\ \mathrm{C} 11-\mathrm{N} 2-\mathrm{C} 7-\mathrm{O} 2 & -177.7(2) \\ \mathrm{C} 11-\mathrm{N} 2-\mathrm{C} 7-\mathrm{C} 8 & 2.2(3) \\ \mathrm{O} 2-\mathrm{C} 7-\mathrm{C} 8-\mathrm{C} 9 & 178.2(2) \\ \mathrm{N} 2-\mathrm{C} 7-\mathrm{C} 8-\mathrm{C} 9 & -1.8(3) \\ \mathrm{C} 7-\mathrm{C} 8-\mathrm{C} 9-\mathrm{C} 10 & 0.6(3) \\ \mathrm{C} 8-\mathrm{C} 9-\mathrm{C} 10-\mathrm{C} 11 & 0.4(3) \\ \mathrm{C} 9-\mathrm{C} 10-\mathrm{C} 11-\mathrm{N} 2 & 0.0(3) \\ \mathrm{C} 9-\mathrm{C} 10-\mathrm{C} 11-\mathrm{C} 12 & -179.9(2) \\ \mathrm{C} 7-\mathrm{N} 2-\mathrm{C} 11-\mathrm{C} 10 & -1.3(3) \\ \mathrm{C} 7-\mathrm{N} 2-\mathrm{C} 11-\mathrm{C} 12 & 178.6(2) \\ \end{array}$

Hydrogen-bond geometry $\left(\AA,{ }^{\circ}\right)$

\begin{tabular}{|c|c|c|c|c|}
\hline$D-\mathrm{H} \cdots A$ & $D-\mathrm{H}$ & $\mathrm{H} \cdots A$ & $D \cdots A$ & $D-\mathrm{H} \cdots A$ \\
\hline $\mathrm{O} 3-\mathrm{H} 1 O \cdots \mathrm{O} 2$ & $0.82(1)$ & $1.88(1)$ & $2.690(2)$ & $175(3)$ \\
\hline $\mathrm{O} 3-\mathrm{H} 2 \mathrm{O} \cdots \mathrm{O} 7$ & $0.81(1)$ & $1.94(1)$ & $2.750(3)$ & $171(3)$ \\
\hline $\mathrm{O} 4-\mathrm{H} 4 O \cdots \mathrm{O} 6^{\mathrm{i}}$ & $0.82(1)$ & $1.97(1)$ & $2.766(3)$ & $166(3)$ \\
\hline $\mathrm{O} 4-\mathrm{H} 3 O \cdots \mathrm{O} 6^{\mathrm{ii}}$ & $0.82(1)$ & $1.99(1)$ & $2.789(3)$ & $169(2)$ \\
\hline $\mathrm{O} 5-\mathrm{H} 5 \mathrm{O} \cdots \mathrm{O} 1$ & $0.82(1)$ & $1.89(1)$ & $2.701(2)$ & $170(3)$ \\
\hline $\mathrm{O} 5-\mathrm{H} 6 O^{\cdots}{ }^{\cdots} 3^{\mathrm{iii}}$ & $0.82(1)$ & $1.94(1)$ & $2.751(3)$ & $169(2)$ \\
\hline $\mathrm{O} 6-\mathrm{H} 7 O^{\cdots} \mathrm{O} 12$ & $0.81(1)$ & $1.94(1)$ & $2.744(3)$ & $173(3)$ \\
\hline $\mathrm{O} 6-\mathrm{H} 8 \mathrm{O} \cdots \mathrm{O}^{\mathrm{iv}}$ & $0.81(1)$ & $1.95(1)$ & $2.760(3)$ & $172(3)$ \\
\hline $\mathrm{O} 7-\mathrm{H} 100 \cdots \mathrm{O} 12^{\mathrm{v}}$ & $0.81(1)$ & $1.94(1)$ & $2.754(2)$ & $174(3)$ \\
\hline $\mathrm{O} 7-\mathrm{H} 90 \cdots \mathrm{O} 11^{\mathrm{vi}}$ & $0.81(1)$ & $1.97(1)$ & $2.774(3)$ & $176(3)$ \\
\hline $\mathrm{O} 8-\mathrm{H} 11 O^{\cdots} \mathrm{O} 11$ & $0.82(1)$ & $2.01(1)$ & $2.823(3)$ & $175(3)$ \\
\hline $\mathrm{O} 8-\mathrm{H} 12 \mathrm{O} \cdots \mathrm{O} 9$ & $0.82(1)$ & $1.96(1)$ & $2.780(3)$ & $177(3)$ \\
\hline $\mathrm{O} 9-\mathrm{H} 13 \mathrm{O}^{\cdots} \mathrm{O} 7$ & $0.82(1)$ & $1.99(1)$ & $2.801(3)$ & $168(3)$ \\
\hline $\mathrm{O} 9-\mathrm{H} 140 \cdots \mathrm{O} 4^{\mathrm{iv}}$ & $0.81(1)$ & $2.02(1)$ & $2.816(3)$ & $166(3)$ \\
\hline $\mathrm{O} 10-\mathrm{H} 16 \mathrm{O}^{\cdots} \mathrm{O} 4$ & $0.82(1)$ & $1.92(1)$ & $2.739(3)$ & $176(3)$ \\
\hline $\mathrm{O} 10-\mathrm{H} 15 \mathrm{O}^{\cdots} \mathrm{O} 5$ & $0.82(1)$ & $1.92(1)$ & $2.736(3)$ & $174(2)$ \\
\hline $\mathrm{O} 11-\mathrm{H} 170 \cdots \mathrm{O} 10^{\mathrm{v}}$ & $0.82(1)$ & $1.94(1)$ & $2.752(2)$ & $173(3)$ \\
\hline $\mathrm{O} 11-\mathrm{H} 18 O \cdots \mathrm{O} 5^{\mathrm{iii}}$ & $0.82(1)$ & $1.93(1)$ & $2.743(2)$ & $175(3)$ \\
\hline $\mathrm{O} 12-\mathrm{H} 190 \cdots \mathrm{O} 3^{\mathrm{vii}}$ & $0.82(1)$ & $1.91(1)$ & $2.726(2)$ & $177(3)$ \\
\hline $\mathrm{O} 12-\mathrm{H} 20 O \cdots \mathrm{O} 10$ & $0.82(1)$ & $1.93(1)$ & $2.743(3)$ & $177(3)$ \\
\hline
\end{tabular}


supporting information

\begin{tabular}{lllll}
$\mathrm{N} 1-\mathrm{H} 1 N \cdots \mathrm{O} 1^{\mathrm{iii}}$ & $0.81(3)$ & $2.00(3)$ & $2.798(3)$ & $171(3)$ \\
$\mathrm{N} 2-\mathrm{H} 2 N \cdots \mathrm{O} 2^{\mathrm{vii}}$ & $0.87(3)$ & $1.92(3)$ & $2.783(3)$ & $175(2)$ \\
\hline
\end{tabular}

Symmetry codes: (i) $x+1, y, z$; (ii) $-x+1,-y,-z$; (iii) $-x+2,-y+1,-z+1$; (iv) $-x+1,-y,-z+1$; (v) $x, y, z+1$; (vi) $x-1, y, z$; (vii) $-x+1,-y+1,-z+1$. 